\title{
A NEW ANGIOCARDIOGRAPHIC SIGN OF PATENT DUCTUS ARTERIOSUS
}

\author{
BY \\ R. H. GOETZ \\ From the Department of Surgical Research, University of Cape Town, and Department of Peripheral Vascular Disease \\ Groote Schuur Hospital, Cape Town, S. Africa
}

Received November 6, 1950

It is generally agreed that the clinical diagnosis of patent ductus arteriosus, although easy in many cases, may be very difficult indeed, particularly in such cases where the typical continuous (fistulous) machinery murmur and the thrill over the pulmonic area are not present. As the diagnosis of patent ductus arteriosus carries with it the practical implication of surgical intervention and as the latter today is a relatively safe and easy procedure, it is important to establish the diagnosis beyond reasonable doubt. Any new sign, therefore, that may help in the positive diagnosis of this abnormality is of interest. The ductus itself is rarely visualized during angiocardiography and the angiocardiographic diagnosis of patent ductus depends entirely on indirect evidence, such as: (1) dilatation of the main or left pulmonary artery; (2) elevation of the main or the left pulmonary artery towards the " isthmus of the aorta "; (3) re-opacification of the pulmonary artery from the aorta; (4) persistance of the dye in the pulmonary artery until such time as the left heart is filled as well; (5) localized dilatation of the descending aorta just beyond the isthmus, and (6) left ventricular dilatation (Steinberg et al., 1943; Donovan et. al., 1943; Chavez et al., 1947; Keele, 1948; Dotter and Steinberg, 1949).

Needles to say, all these signs are not present in all cases. According to Donovan et. al., 31 out of 50 proved cases of patent ductus arteriosus have had dilatation of the pulmonary artery, and in 25 per cent of the cases investigated by Steinberg et al. the dilatation of the pulmonary artery was absent; 26 of their 27 cases showed the dilatation of the aorta described above, which thus, appears to be a more constant feature. However, we have had difficulty in demonstrating this sign in our cases. The re-opacification of the pulmonary artery as the diodone passes through the aorta may also be difficult to see, as the diodone is often too diluted at this stage and on mixing with the blood in the pulmonary artery it may be practically invisible. This is particularly the case if the ductus is a narrow channel.

The direction of the blood flow in a patent ductus is, with very rare exceptions, from the aorta to the pulmonary artery. This has been proved at operations and by cardiac catheterization. Eppinger and Burwell (1941) have calculated that the volume of blood shunted from the aorta to the pulmonary artery in three of their cases measured between 45 and 77 per cent of the stroke volume of the left ventricle. It is by this " injection " of blood into the pulmonary artery from the aorta that the sign to be described is produced.

The sign consists of a defect in the outline of the main pulmonary artery and is seen at a time when the contrast medium is passing through the pulmonary artery, that is, two to three seconds after the injection of the diodone. As the blood from the aorta does not contain any diodone at that time, the admixture of blood from the aorta via the patent ductus with the blood in the pulmonary artery containing the diodone shows up on the film as a filling defect. This defect is clearly seen in Fig. 1 and 2, and has been seen by us in the last four consecutive cases of patent ductus arteriosus. 


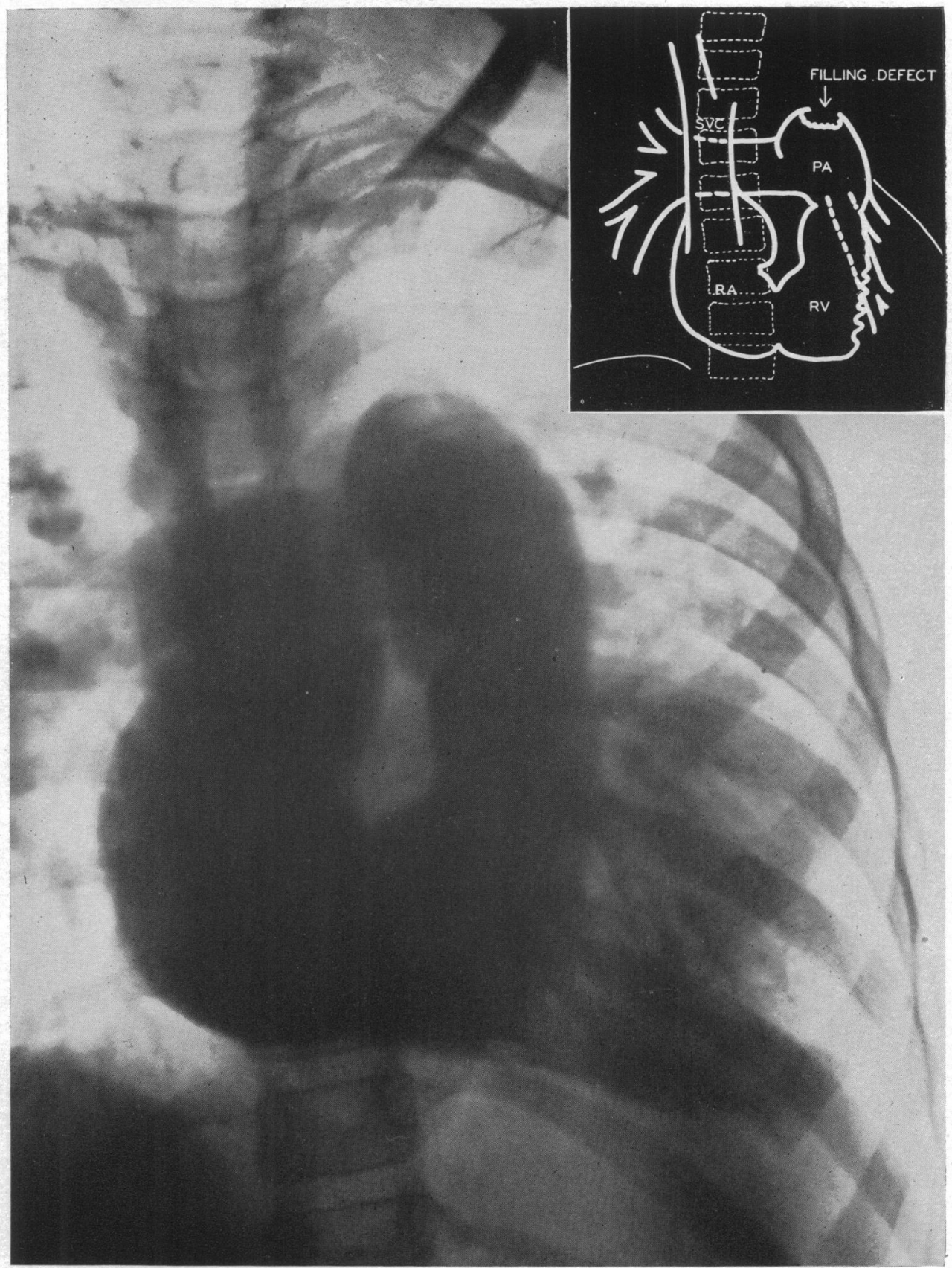

FIG. 1.-Antero-posterior view at $3 \mathrm{sec}$., showing outline of right auricle, ventricle, and pulmonary artery. Note the defect in the filling of the pulmonary artery. Proved case of patent ductus arteriosus. 


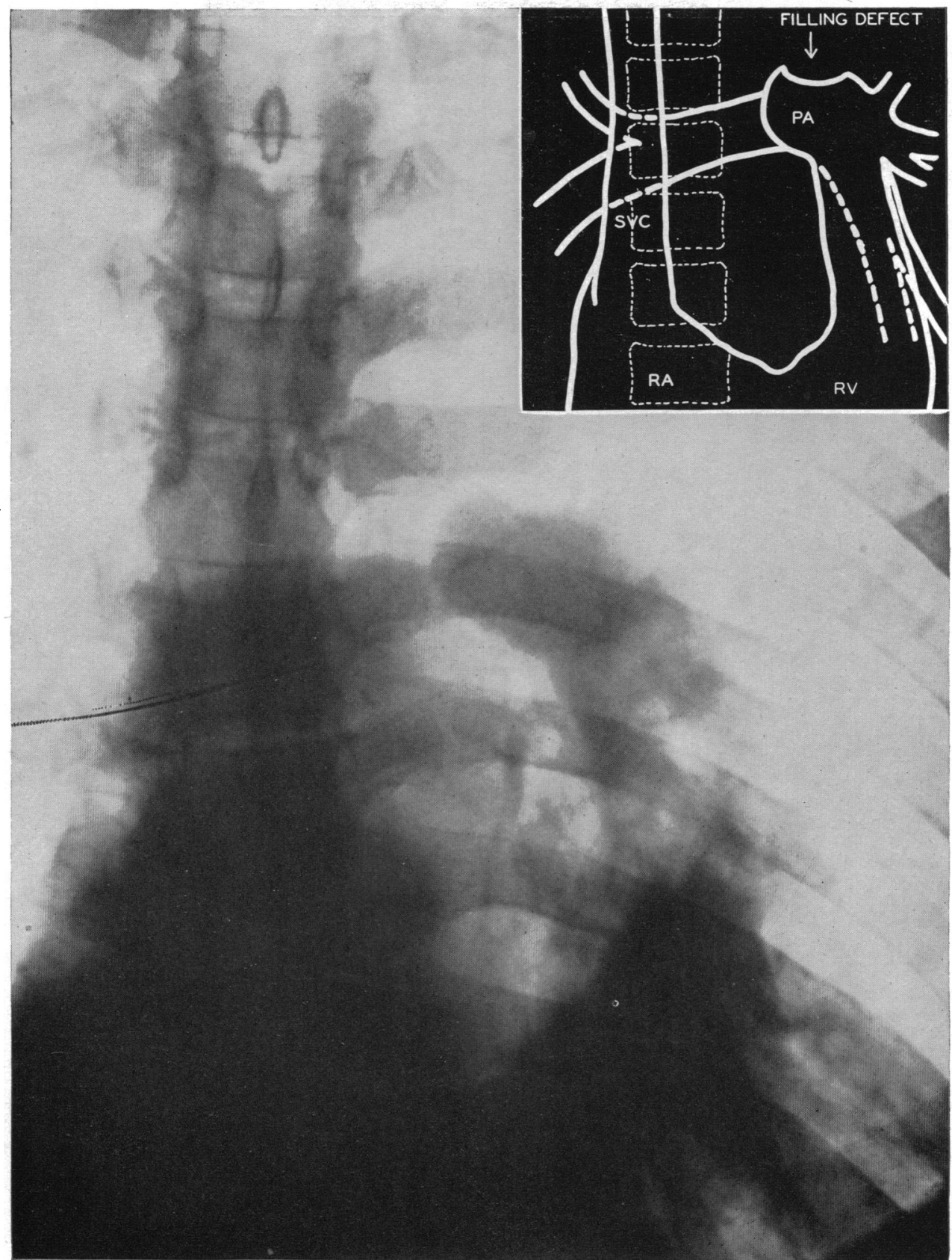

FIG. 2.-Antero-posterior view taken at 2 sec., showing clearly superior vena cava, right auricle, right ventricle, and main pulmonary artery filled. The defective filling in the elevated and dilated main pulmonary artery is clearly seen. 


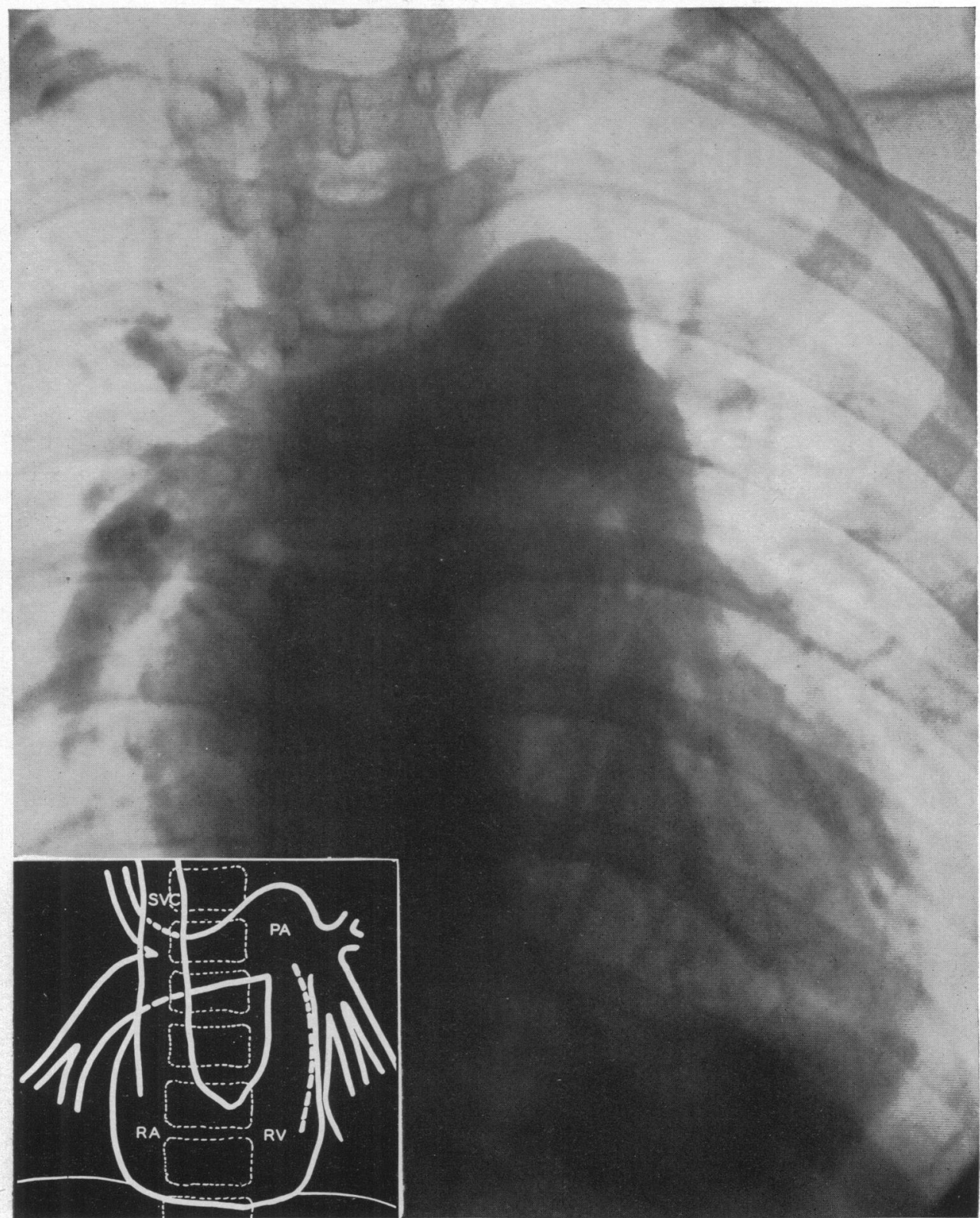

Fig. 3.-Antero-posterior view taken at $3 \mathrm{sec}$. to show dilated main pulmonary artery in a case of infundibular stenosis. Note the clear outline of the main pulmonary artery.

Two of these were subjected to operation when the diagnosis was confirmed.* The dilatation and elevation of the pulmonary artery as first observed by Steinberg et al. is also clearly seen in Fig. 1 .

The filling defect, although usually seen in both views, may be more definite in the left anterior oblique position. It may not be present on all plates in which the pulmonary artery is filled,

* These cases were operated on by Mr. Walter Phillips. 
presumably depending on the phase of the cardiac cycle at which the plate was taken. It is therefore advisable to take more than one exposure during the time at which the diodone is passing through the pulmonary artery. In our experience, the visualization of this filling defect may be of considerable importance in making a positive diagnosis of patent ductus arteriosus in such cases where the opening is rather small and where consequently, both the dilatation of the pulmonary artery and the re-opacification of the pulmonary artery are absent. Fig. 3 is reproduced to show a marked dilatation of the main pulmonary artery in a case other than patent ductus, and was obtained in a case of infundibular stenosis with some post-stenotic dilatation. The sharp outline of the pulmonary artery in this case is clearly seen and contrasts well with the defect in the two cases with patent ductus arteriosus.

\section{SUMMARY}

A new sign in the angiocardiographic diagnosis of patent ductus arteriosus is demonstrated. It consists of a defect in the homogeneous filling with the opaque material of the main pulmonary artery. It is brought about by the injection of blood from the aorta through the ductus diluting the opaque medium as it passes through the pulmonary artery.

I am most grateful to my colleagues in the Department of Medicine, Prof. F. Forman, Prof. F. Brock, and Dr. S. Michelson for referring the cases used in this study. I am grateful for the assistance received from Dr. A. Katz and the staff of the X-ray Department.

\section{REFERENCES}

Chavez, I., Dorbecker, N., and Celis, A. (1947). Amer. Heart J., 33, 560.

Donovan, M. S., Neuhauser, E. B. D., and Sosman. M. C. (1943). Amer. J. Roentgen. Radiother., $50,293$.

Dotter, C. T., and Steinberg, I. (1949). J. Amer. med. Assoc., 139, 566.

Eppinger, E. C., and Burwell, C. S. (1941). Clin. Invest., 20, 127.

Keele, K. D. (1948). Brit. J. Radiol., 21, 380.

Steinberg, M. F., Grishman, A., and Sussman, M. L. (1943). Amer. J. Roentgen. Radiother., 50, 306. 